\title{
Negative universality results for graphs
}

\author{
by
}

\section{S.-D. Friedman and K. Thompson (Wien)}

\begin{abstract}
It is shown that in many forcing models there is no universal graph at the successors of regular cardinals. The proof, which is similar to the well-known proof for Cohen forcing, is extended to show that it is consistent to have no universal graph at the successor of a singular cardinal, and in particular at $\aleph_{\omega+1}$. Previously, little was known about universality at the successors of singulars. Analogous results show it is consistent not just that there is no single graph which embeds the rest, but that it takes the maximal number $\left(2^{\lambda}\right.$ for graphs of size $\left.\lambda\right)$ to embed the rest.
\end{abstract}

1. Introduction. A universal model for graphs of size $\lambda$ (also called $\lambda$-graphs) is one which embeds all other $\lambda$-graphs. Here we consider graph embeddings which are injective maps whose image as an induced subgraph of the range is isomorphic to the graph in the domain. Note that if we consider embeddings which only preserve edges, the complete graph on $\lambda$ is always universal for $\lambda$-graphs for any cardinal $\lambda$.

Since the theory of graphs can be defined in first-order logic and is countable, assuming the generalised continuum hypothesis $(\mathrm{GCH})$ there are universal models in every uncountable cardinal. However, the theory is unstable, and therefore model-theoretically it is not clear what happens when GCH fails. In particular, the question is in which models of $\lambda^{<\lambda}>\lambda$ there are universal $\lambda$-graphs.

Shelah in [12] showed it is consistent that $2^{\lambda}=\lambda^{++}$for $\lambda$ regular and there is a universal model for $\lambda^{+}$-graphs. The negative result is also consistent.

TheOREM 1.1 (Shelah). Starting with a model in which $2^{\lambda}=\lambda^{+}$, and forcing $\lambda^{++}$-many Cohen subsets of $\lambda$ for $\lambda$ regular, results in a model where there is no universal graph of size $\lambda^{+}$.

2010 Mathematics Subject Classification: Primary 03E75, 03E35; Secondary 03E05, 03E55. Key words and phrases: graphs, triangle-free graphs, bipartite graphs, universal models, successors of singular cardinals. 
The proof for the above result can be found in 8 and involves an interesting use of intermediate models. As parts of the proof will be used in later sections, the whole proof will be given in a more general setting in Section 2 for the convenience of the reader.

For a survey of other universality results concerning graphs, see [14.

In Section 2 Shelah's negative universality result is extended to a wide variety of forcings, including $\lambda$-proper product forcings (for various definitions of $\lambda$-proper) such as products of $\lambda$-Sacks forcings. These results are used in later sections to show non-existence of universal graphs in the context of measurable cardinals. However, this is also an interesting result in its own right: The abundance of models to which this result can be applied underscores the difficulty of finding models in which there are universal graphs in the absence of GCH. In particular, it is still open whether there can be a model of a failure of $\mathrm{CH}$ in which there is a universal triangle-free graph at $\aleph_{1}$ (see [11] for more details). Corollary 2.7 below gives more evidence that this is indeed a difficult question.

On the other hand, the forcings which may be applied in these contexts are clearly limited to products. In [13], a $\lambda^{++}$-length $(<\lambda)$-support iteration which has the $\lambda^{+}$-cc is used to obtain a model where there is a universal graph at $\lambda^{+}$and $2^{\lambda}=\lambda^{++}$. This shows that even "nice, gentle" iterations which add subsets of $\lambda$ at each step cannot generally be used to show negative universality results.

Shelah's original result for Cohen forcing can also be extended to give a global universality result for graphs:

COROLlary 1.2. There is a model of ZFC such that at every regular cardinal $\lambda$, there is no universal $\lambda^{+}$-graph, and at limit cardinals $\lambda$, there is a universal $\lambda$-graph.

Such a model can be built using an Easton product forcing adding $\lambda^{++}$ many Cohen subsets of $\lambda$ for each regular $\lambda$ (see e.g. [6] for this forcing). Adding only $\lambda^{++}$-many ensures that $\lambda^{<\lambda}=\lambda$ continues to hold at limit cardinals.

What is missing from this picture is a non-trivial result at the successors of singular (strong limit) cardinals. This is a problem as singular cardinal combinatorics is a notoriously difficult field. In particular, a failure of the Singular Cardinal Hypothesis $\left(2^{\lambda}>\lambda^{+}\right.$for singular $\left.\lambda\right)$ requires large cardinals (see e.g. [4]).

In Section 3 the cases where $\lambda$ is measurable or singular are considered. In both cases it is assumed that a $(\lambda+2)$-hypermeasurable cardinal (see Definition 3.1) exists and in the latter case Prikry forcing is used to singularise $\lambda$. Note that by [5] these results, which include raising the value of $2^{\lambda}$, are near-optimal in consistency strength. 
To our knowledge, the only known universality result at the successor of a singular strong limit cardinal $\lambda$ when $2^{\lambda}>\lambda^{+}$is given by Džamonja and Shelah in [1], where they show that it is consistent relative to a supercompact cardinal that there is a small universal family for $\lambda^{+}$-graphs where $\lambda$ has cofinality $\aleph_{0}$. A (small) universal family for $\lambda^{+}$-graphs is a set of $\lambda^{+}$-graphs (of size $<2^{\lambda^{+}}$) which, taken together, embed all $\lambda^{+}$-graphs. The minimal size of a universal family is called the complexity.

In Section 5 the results in this paper are extended to show that the forcings specified in Section 2 produce models in which there is also no small universal family of $\lambda^{+}$-graphs, thus the complexity is maximal, namely $2^{\lambda^{+}}$.

Section 4 is devoted to the specific case of universal graphs at $\aleph_{\omega+1}$. The techniques from Section 3 are modified from standard Prikry forcing to the forcing in [3] which collapses a hypermeasurable cardinal to $\aleph_{\omega}$.

When ordering forcing conditions, $p \leq q$ will mean that $p$ is stronger than $q$. If $p$ is a forcing condition and $p$ forces either $\sigma$ or $\neg \sigma$ we say that $p$ decides $\sigma$ and write $p \| \sigma$. If $P=\left\langle P_{\alpha}: \alpha<\alpha^{*}\right\rangle$ is a product forcing or an iteration and $G$ is $P$-generic, we denote by $P(<\alpha)$ and $G(<\alpha)$ the restriction of the forcing and generic, respectively, to the first $\alpha$ coordinates of the forcing. If $G$ is $P$-generic and $G$ can be coded as a subset of ordinals $A$, we abuse notation and call $A$ generic. If $G$ is $P$-generic and $\underset{\tilde{G}}{B}$ is a $P$-name for a set, then we denote by $B^{G}$ the interpretation of $\underset{\sim}{B}$ by $G$.

We use a number of standard forcings which will not be defined in this paper. Definitions and properties of Cohen, Sacks, Prikry and Levy collapse forcings can be found in [6]. For Sacks on uncountable cardinals, see [7] or [2].

\section{Many forcings destroy universality for graphs at successors} of regulars. In this section, let $\lambda$ be an infinite regular cardinal.

Definition 2.1. We say that a forcing $P$ has the $\lambda$-covering property if whenever $p \in P$ and $\underset{\sim}{B}$ is a $P$-name for a subset of the ground model such that $p \Vdash \underset{\sim}{B}$ has size $\lambda$, there exists $q$ extending $p$ and $C$ in the ground model of size $\lambda$ such that $q \Vdash \underset{\sim}{B} \subseteq C$.

Forcings that have the $\lambda$-covering property preserve $\lambda^{+}$.

TheOREM 2.2. Let $V \vDash 2^{<\lambda}=\lambda$ and let $P$ be a product forcing $\left\langle P_{\alpha}: \alpha<\alpha^{*}\right\rangle$ with $\delta$-support for some $\delta \leq \lambda$ such that $\operatorname{cf}\left(\alpha^{*}\right) \geq \lambda^{++}$, and $P$ has the $\lambda$-covering property such that at any component $\alpha$ the $P_{\alpha}$-generic is $A_{\alpha} \subset \lambda$. Then in $V^{P}$ there is no universal graph of size $\lambda^{+}$.

Proof. Let $P$ be as in the statement of the theorem and let $G$ be $P$ generic over $V$. For the sake of contradiction, assume that $H^{*} \in V[G]$ is a universal graph of size $\lambda^{+}$. 
Lemma 2.3. There is $\alpha<\lambda^{++}$such that $H^{*} \in V_{\alpha}:=V[G(<\alpha)]$.

Proof. We may write $H^{*}$ as $\left(\lambda^{+}, E_{H^{*}}\right)$ where $\lambda^{+}$is the universe and $E_{H^{*}} \subseteq \lambda^{+} \times \lambda^{+}$is the edge relation. The edge relation can be coded as a subset of $\lambda^{+}$, say $A$, so we will show that there exists $\alpha$ such that $A \in V_{\alpha}$. Let $A$ be the canonical $P$-name for $A$.

For each $i<\lambda^{+}$let $Y_{i}=\{p \in P: p \| i \in \underset{\sim}{A}\}$, which is dense in $P$. Let $X_{i} \subseteq Y_{i}$ be a maximal antichain in $P$. Let $p_{i} \in X_{i} \cap G$ and let $\alpha_{i}$ be the supremum of the support of $p_{i}$, which is bounded in $\alpha^{*}$. This induces a function $g: \lambda^{+} \rightarrow \alpha^{*}$ given by $g(i)=\alpha_{i}$, which, by the fact that $\operatorname{cf}(\alpha) \geq$ $\lambda^{++}$, must be bounded in $\alpha^{*}$. Let $\alpha$ be the supremum of the range of $g$. The name $\underset{\sim}{A}$ depends only on components $<\alpha$ and thus $A \in V_{\alpha}$.

As $\alpha^{*}$ has cofinality at least $\lambda^{++}$, there are at least $\lambda^{++}$-many generic sets $A_{\beta}$ for $\beta>\alpha$ which are in $V[G] \backslash V_{\alpha}$.

We will construct a graph $H \in V[G]$ of size $\lambda^{+}$such that there is no embedding of $H$ into $H^{*}$. Since this is the same construction for all proofs in this paper, we give it here so that we may refer to it later.

Counterexample Construction 2.4. Let $H$ have universe $\lambda \cup Y$ where $\min (Y)>\lambda$. Define $E_{H}$ by letting $(\gamma, \beta)$ be an edge in $H$ if and only if $\gamma \in Y$ and $\beta \in A_{\gamma}$, or symmetrically, $\beta \in Y$ and $\gamma \in A_{\beta}$.

In this proof, let $Y=\left[\alpha, \alpha+\lambda^{+}\right)$. By mutual genericity we have $A_{\gamma} \notin V_{\alpha}$ for all $\gamma \geq \alpha$.

Lemma 2.5. For any $B \in V[G]$ such that $B$ has size $\lambda$ there exists $V^{\prime}$ such that $V_{\alpha} \subseteq V^{\prime} \subseteq V[G]$ and there are at most $\lambda$-many of the $A_{\gamma}$ in $V^{\prime} \backslash V_{\alpha}$ for $\gamma \in Y$ and $B \in V^{\prime}$.

Proof. As above, for each $i<\lambda$ we choose $X_{i}$, a maximal antichain of conditions deciding whether $i \in B$. Let $Z=\left\{p_{i}:\left\{p_{i}\right\}=X_{i} \cap G\right\}$ and let $\underset{\sim}{Z}$ be a $P$-name for $Z$. By $\lambda$-covering, given $\underset{\sim}{Z}$ and a condition $p \in P$ there exists $C$ of size $\lambda$ in $V$ and $q \leq p$ such that $q \Vdash \underset{\sim}{Z} \subseteq C$. As $P$ is a product, we can consider only those components which are in the support of $q$ and in each $p_{i}$, of which there are at most $\lambda$-many. Let $D=\left\{A_{\beta}: \beta \in \operatorname{supp}(q) \cup \bigcup_{i<\lambda} \operatorname{supp}\left(p_{i}\right)\right\}$ and let $V^{\prime}=V_{\alpha}[D]$. Then $V^{\prime}$ has the desired properties.

By the universality of $H^{*}$ there exists an embedding $f: H \rightarrow H^{*}$. The object $f\left\lceil\lambda\right.$ has size $\lambda$ so there exists $V^{\prime}$ as in the lemma such that $f\left\lceil\lambda \in V^{\prime}\right.$.

Lemma 2.6. For all $\gamma \in Y, A_{\gamma}$ can be recovered from $f\left\lceil\gamma\right.$ and $H^{*}$ in $V^{\prime}$.

Proof. For $y \in H^{*}$ let $H_{y}^{*}=\left\{x \in \lambda:(f(x), y) \in E_{H^{*}}\right\}$. As $f\left\lceil\lambda \in V^{\prime}\right.$ and $H^{*} \in V_{\alpha}$, for all $y \in H^{*}$ we have $H_{y}^{*} \in V^{\prime}$.

Set $y=f(\gamma)$. Then $B_{y}^{*}:=\left\{x:(f(x), f(\gamma)) \in E_{H^{*}}\right\}$ is the same as $\{x:(x, \gamma)$ is an edge in $H\}$ by the edge preservation of $f$. 
Thus, by definition of $H$, either $x \in Y$ and $\gamma \in A_{x}$, or $\gamma \in Y$ and $x \in A_{\gamma}$. By definition, $A_{x} \subseteq \lambda$ so it cannot be the case that $\gamma \in A_{x}$. Therefore, $B_{y}^{*}=\left\{x: x \in A_{\gamma}\right\}=A_{\gamma}$.

Now $B_{y}^{*} \cap \lambda=H_{y}^{*} \in V^{\prime}$ and thus $A_{\gamma} \in V^{\prime}$.

However, by the definition of $Y$ and $V^{\prime}$ there exists $\gamma \in Y$ such that $A_{\gamma} \notin V^{\prime}$, contradiction. This finishes the proof of Theorem 2.2.

Corollary 2.7. If $P$ is as in Theorem 2.2 then in $V^{P}$ there is no universal

- graph of size $\lambda^{+}$which omits complete subgraphs of size $\theta$ for any $\theta>2$,

- bipartite graph of size $\lambda^{+}$.

Note that in the construction of the graph $H$, for any $\gamma \in Y$ and $\beta \in A_{\gamma}$ we have $\beta \in \lambda$. So, $(\gamma, \beta)$ being an edge in $H$ implies that $\gamma \geq \lambda$ and $\beta<\lambda$ or vice versa. This gives no triangles in $H$, and indeed, no complete subgraphs of any larger size. The sets $\{\alpha: \alpha<\lambda\}$ and $\{\gamma: \gamma \in Y\}$ form the partition needed for a bipartite graph.

Similar corollaries will also hold for triangle-free graphs and bipartite graphs of size $\kappa^{+}$where $\kappa, P$ are as in Theorems $3.2,3.3,4.1$ and 5.1 .

\section{Non-existence of universal graphs at successors of measur-} ables and singulars. In this section, for an inaccessible cardinal $\alpha$, let $\operatorname{Sacks}\left(\alpha, \alpha^{++}\right)$be the $\alpha$-support product of $\alpha$-Sacks forcing of length $\alpha^{++}$. A condition in $\alpha$-Sacks forcing is a subset of $<^{<} 2$ which is a closed tree for which there is a club of levels such that a node of the tree splits if and only if the height of the node is in the club (called the splitting levels). The $\beta$ th

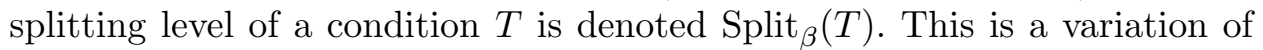
the forcing defined in [7] which is used in [2].

Definition 3.1. A $(\kappa+\alpha)$-hypermeasurable cardinal $\kappa$ is one for which there exists an embedding $j: V \rightarrow M$ such that $\operatorname{crit}(j)=\kappa, M$ is closed under $\kappa$-sequences and $H\left(\kappa^{+\alpha}\right)^{V}=H\left(\kappa^{+\alpha}\right)^{M}$.

Theorem 3.2. Assume $V \vDash G C H$ and $\kappa$ is a $(\kappa+2)$-hypermeasurable cardinal. There is a forcing $\mathbb{P}$ such that in $V^{P}$ there is no universal $\kappa^{+}$-graph, $\kappa$ remains measurable and $2^{\kappa}>\kappa^{+}$.

Proof. Let $\mathbb{P}=\left\langle\mathbb{P}_{\alpha}: \alpha \leq \kappa\right\rangle$ be an Easton-support iteration which is trivial except when $\alpha$ is inaccessible. For such $\alpha$ let $\mathbb{P}_{\alpha}=\operatorname{Sacks}\left(\alpha, \alpha^{++}\right)$.

By arguments in [2], as we assumed that $\kappa$ was $(\kappa+2)$-hypermeasurable, this forcing preserves $\kappa$ as a measurable cardinal and sets $2^{\kappa}=\kappa^{++}$. Also $\mathbb{P}_{\kappa}=$ $\operatorname{Sacks}\left(\kappa, \kappa^{++}\right)$preserves $\kappa^{++}$and has the $\kappa$-covering property in $V[G(<\kappa)]$ 
as $2^{\kappa}=\kappa^{+}$and $2^{<\kappa}=\kappa$ in this model. Thus, by Theorem 2.2 applied in the model $V[G(<\kappa)]$, there is no universal graph after forcing with $\mathbb{P}_{\kappa}$.

Theorem 3.3. Assume $V \vDash G C H$ and $\kappa$ is a $(\kappa+2)$-hypermeasurable cardinal. There is a forcing $\mathbb{P}$ such that in $V^{P}$ there is no universal $\kappa^{+}$-graph, $\kappa$ is singular and $2^{\kappa}>\kappa^{+}$.

Proof. Let $\mathbb{P}=\left\langle\mathbb{P}_{\alpha}: \alpha \leq \kappa\right\rangle$ be an Easton-support iteration which is trivial except when $\alpha$ is inaccessible. For $\alpha<\kappa$ inaccessible let $\mathbb{P}_{\alpha}=$ $\operatorname{Sacks}\left(\alpha, \alpha^{++}\right)$. Let $\mathbb{P}_{\kappa}=\operatorname{Sacks}\left(\kappa, \kappa^{++}\right) * \operatorname{Pr}(U)$ where $\operatorname{Pr}(U)$ is Prikry forcing at $\kappa$ using some normal measure $U$ on $\kappa$ whose $\operatorname{Sacks}\left(\kappa, \kappa^{++}\right)$-name is $\underset{\sim}{U}$.

Let $G_{\kappa}^{S}$ be generic for $\operatorname{Sacks}\left(\kappa, \kappa^{++}\right)$over $V[G(<\kappa)]=V_{0}$. For $\alpha \leq \kappa^{++}$ we denote $V\left[G(<\kappa), G_{\kappa}^{S}(<\alpha)\right]$ by $V_{\alpha}$. For each component $\gamma$ of $\operatorname{Sacks}\left(\kappa, \kappa^{++}\right)$, we denote by $A_{\gamma}$ the subset of $\kappa$ which codes the generic for that component. Let $U \in V_{\kappa^{++}}$be the normal measure on $\kappa$ which is used to define the Prikry forcing.

By arguments in [2], this forcing preserves cardinals and sets $2^{\kappa}=\kappa^{++}$. The Prikry forcing is well-defined, since $\kappa$ remains measurable after forcing over $V_{0}$ with $\operatorname{Sacks}\left(\kappa, \kappa^{++}\right)$. It remains to fold in the Prikry forcing to the argument that no universal graph exists in the extension. In order to do this, we must be able to restrict the Sacks product at $\kappa$, but still be able to define the Prikry generic. We state a general lemma to this effect.

Lemma 3.4. Suppose that $V$ is a model of $2^{\kappa}=\kappa^{+}$. Let $P$ be $a \leq \kappa-$ support product of forcings $P_{i}$ for $i<\kappa^{++}$with $\left|P_{i}\right| \leq \kappa^{+}$such that $P$ has the $\kappa$-covering property. Suppose that $G$ is $P$-generic and that $U$ is a normal measure on $\kappa$ in $V[G]$. Then

$$
T=\left\{\alpha<\kappa^{++}: \operatorname{cf}(\alpha)=\kappa^{+} \text {and } U \cap V[G(<\alpha)] \in V[G(<\alpha)]\right\}
$$

is a stationary subset of $\kappa^{++}$.

Proof. Let $\underset{\sim}{U}$ be a $P$-name for $U$ and let $N \in V$ be an elementary submodel of some large $H(\theta)$ which has size $\kappa^{+}$, is closed under $\kappa$-sequences, and has $P, \underset{\sim}{U}$ as elements. By closure under $\kappa$-sequences we see that $\alpha=$ $N \cap \kappa^{++}$has cofinality $\kappa^{+}$. In fact, since $\kappa^{+} \subseteq N$, it follows that $H\left(\kappa^{++}\right) \cap N$ is transitive. Note that the set of such $\alpha$ is stationary in $\kappa^{++} \cap \operatorname{cof}\left(\kappa^{+}\right)$.

We may assume that each $P_{i}$ has universe $\kappa^{+}$. This together with the $\kappa$-support of $P$ and the closure of $N$ under $\kappa$-sequences implies that $P \cap N$ is precisely $P(<\alpha)$. Let $G$ be $P$-generic over $V$. Since $P$ has the $\kappa^{++}$-cc and by the transitivity of $H\left(\kappa^{++}\right) \cap N$, if $A \in N$ is a maximal antichain in $P$, then in fact $A \subseteq N$. Thus $G \cap A \cap N \neq \emptyset$; so $G \cap N$ is $N$-generic. In fact, since $P \cap N=P(<\alpha)$ we deduce that $G \cap N=G(<\alpha)$. Therefore $N[G(<\alpha)]$ is an elementary submodel of $H(\theta)[G]$.

Let $B$ be any subset of $\kappa$ in $V[G(<\alpha)]$. Let $\underset{\sim}{B}$ be a $P(<\alpha)$-name for $B$ and let $p \in P(<\alpha)$ force that $\underset{\sim}{B} \subseteq \kappa$. For each $i<\kappa$ let $D_{i}=\{r \leq p: r \| i \in \underset{\sim}{B}\}$. 
As $D_{i}$ is dense in $P(<\alpha)$ we may find $X_{i} \subseteq D_{i}$, a maximal antichain in $P(<\alpha)$. Define a function $f: \kappa \rightarrow P(<\alpha)$ by $\{f(i)\}=G \cap X_{i}$ for each $i<\kappa$. Thus, $p \Vdash\{\underset{\sim}{f}(i)\}=G \cap X_{i}$ where $\underset{\sim}{f}$ is the canonical $P(<\alpha)$-name for $f$.

By the $\kappa$-covering of $P(<\alpha)$ in $N$, there is $q \leq p$ and $C \in N$ such that $|C|=\kappa$ and $q \Vdash \operatorname{ran}(\underset{\sim}{f}) \subseteq C$. Now $C$ may be used to define a name $B_{\sim}^{\prime}$ in $N$ so that $q \Vdash \underset{\sim}{B}=B_{\sim}^{\prime}$. It follows by elementarity that $U^{G} \cap N[G(<\alpha)]$ equals $U^{G(<\alpha)}$ and belongs to $N[G(<\alpha)]$, therefore to $V[G(<\alpha)]$, as desired.

For $\alpha \in T$ it is clear that $U \cap V_{\alpha}$ is a normal ultrafilter in $V_{\alpha}$ so we may define the Prikry forcing $\operatorname{Pr}\left(U \cap V_{\alpha}\right)$ in $V_{\alpha}$. Let $C$ be the Prikry generic $\omega$-sequence over $V_{\kappa^{++}}$. For $\alpha \in T$ we see that $C$ is also the generic $\omega$-sequence for $\operatorname{Pr}\left(U \cap V_{\alpha}\right)$ over $V_{\alpha}$. This is because of a result of Mathias (see e.g. [6, Theorem 21.14]) which characterises a Prikry sequence $C$ as an $\omega$-length subset of $\kappa$ for which for every $X \in U, C \backslash X$ is finite.

Assume that $H^{*} \in V_{\kappa^{++}}[C]$ is a universal graph of size $\kappa^{+}$and let $\alpha^{*} \in T$ be such that $H^{*} \in V_{\alpha^{*}}[C]$. Such an $\alpha$ may be found as in Lemma 2.3 .

We may construct a graph $H \in V_{\kappa^{+}+}$of size $\kappa^{+}$as in Counterexample Construction 2.4. Let $Y \subseteq \kappa^{++}$of size $\kappa^{+}$be such that $\min (Y)>\alpha^{*}$ and $Y \cap T$ is cofinal in $\sup (Y)$ and has cofinality $\kappa^{+}$.

By the universality of $H^{*}$ there exists an embedding $f: H \rightarrow H^{*}$. The object $f\left\lceil\kappa\right.$ has size $\kappa$ so we would like to find $V^{\prime}$ in a similar way to Lemma 2.5 so that $f\left\lceil\kappa \in V^{\prime}\right.$.

Lemma 3.5. For any $B \in V_{\kappa^{++}}[C]$ such that $B$ has size $\kappa$ there exists $V^{\prime}$ such that $V_{\alpha^{*}}[C] \subseteq V^{\prime} \subseteq V_{\kappa^{++}}[C]$, and $X \subseteq \kappa^{++}$with $|X| \leq \kappa$ such that $V^{\prime}=V_{\alpha^{*}}\left[C, G_{\kappa}^{S} \uparrow X\right]$ and $B \in V^{\prime}$.

Proof. For each $i<\kappa$ we choose $X_{i}$, a maximal antichain of conditions in $\mathbb{P}_{\kappa}$ deciding whether $i \in B$. Let $Z=\left\{p_{i}:\left\{p_{i}\right\}=X_{i} \cap G\right\}$ and let $\underset{\sim}{Z}$ be a $\mathbb{P}_{\kappa}$-name for $Z$. By $\kappa$-covering of $\mathbb{P}_{\kappa}$, given $\underset{\sim}{Z}$ and a condition $p \in \mathbb{P}_{\kappa}$ there

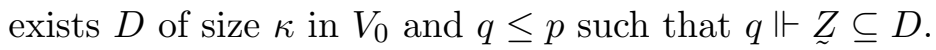

For all $p \in \mathbb{P}_{\kappa}$, the condition $p$ can be written as $\left(p^{S}, p^{\mathrm{Pr}}\right)$ where $p^{S}$ is a condition in $\operatorname{Sacks}\left(\kappa, \kappa^{++}\right)$and $p^{\mathrm{Pr}}$ is forced by $p^{S}$ to be a condition in $\operatorname{Pr}(\underset{\sim}{U})$. Let $X=\left\{\beta: \beta \in \operatorname{supp}\left(q^{\tilde{S}}\right) \cup \bigcup_{i<\kappa} \operatorname{supp}\left(p_{i}^{S}\right)\right\}$. Then $|X|=\kappa$ and $V^{\prime}=V_{\alpha^{*}}\left[C, G_{\kappa}^{S}\lceil X]\right.$ has the desired properties.

We want to see that there is $\gamma \in Y$ such that $A_{\gamma} \notin V^{\prime}$.

Lemma 3.6. There exists $\beta \in Y$ with $\beta \notin X$ such that $A_{\beta} \notin V^{\prime}$.

Proof. First assume there is $\beta \in Y$ such that $\beta>\sup (X)$ and choose one such that there is a $\gamma \in Y \cap T$ with $\sup (X)<\gamma<\beta$. This is possible by assumption on $Y$. Since the $\kappa$-Sacks generic functions are "mutually generic", it must be the case that $A_{\beta} \in V_{\gamma}[C]$ and $A_{\beta} \notin V_{\gamma}$. 
However, it cannot be the case that $V_{\gamma}\left[A_{\beta}\right] \subseteq V_{\gamma}[C]$, as the former contains a club in $\kappa^{+}$which does not contain a club in $V_{\gamma}$, and in the latter model, as Prikry forcing is $\kappa^{+}$-cc, all clubs in $\kappa^{+}$contain clubs in $V_{\gamma}$. To prove the former, we give a general claim:

Claim 3.7. Let $V$ be a model of $2^{\kappa}=\kappa^{+}$and let $S$ be $\kappa$-Sacks forcing. In $V^{S}$ there exists a club $C$ in $\kappa^{+}$such that there is no club $D \in V$ such that $D \subseteq C$.

Proof. Write the $H\left(\kappa^{+}\right)$of $V$ as $L[A]$ where $A \subseteq \kappa^{+}$. Let $\underset{\sim}{A}{ }^{S} \in{ }^{\kappa} 2$ be an $S$-name for the $\kappa$-sequence derived from the $S$-generic.

Define a function $R$ with domain $S \times{ }^{\kappa} 2$ such that

$$
R(T, B)=\left\{\beta<\kappa: B\left(\operatorname{Split}_{2 \cdot \beta}(T)\right)=0\right\} .
$$

Let $C \in V^{S}$ be the set of all $\alpha<\kappa^{+}$such that for all $T \in L_{\alpha}[A] \cap S$ either $R\left(T, A^{S}\right) \in L_{\alpha}[A]$ or $R\left(T, A^{S}\right) \notin V$. For a given $A^{S}$ in the extension, $C$ is a club.

Now we will work in $V$, so let $\underset{\sim}{C}$ be an $S$-name for $C$. Let $X \subseteq \kappa^{+}$be any unbounded subset in $V$ and let $T \in S$ be any condition. Choose $\alpha \in X$ such that $T \in L_{\alpha}[A]$. Prune the tree $T$ on each $\operatorname{Split}_{2 \cdot \beta}(T)$ to obtain $T^{*}$ such that for every branch $B^{*}$ of $T^{*}$ we have $R\left(T, B^{*}\right) \in V \backslash L_{\alpha}[A]$. Thus, $T^{*} \Vdash \alpha \notin C$.

Now let $X$ be such that there is $x \in X$ with $x \geq \sup (Y)$. We would like to rearrange the components of the Sacks product so that we may reduce to the first case. We will do this via an automorphism $\pi: \operatorname{Sacks}\left(\kappa, \kappa^{++}\right) \rightarrow$ $\operatorname{Sacks}\left(\kappa, \kappa^{++}\right)$. Such a map induces an automorphism of $\operatorname{Sacks}\left(\kappa, \kappa^{++}\right)$names, say $\pi^{\prime}$. Note that if $\tau$ is a canonical $\operatorname{Sacks}\left(\kappa, \kappa^{++}\right)$-name for a ground model element then $\pi^{\prime}(\tau)=\tau$. This in turn induces an automorphism of $\operatorname{Sacks}\left(\kappa, \kappa^{++}\right) * \operatorname{Pr}(\underset{\sim}{U})$ as follows:

$$
\pi^{\prime \prime}:(p, \underset{\sim}{q}) \mapsto\left(\pi(p), \pi^{\prime}(\underset{\sim}{q})\right) .
$$

With such an automorphism we have the following fact (see e.g. 9]):

FACT 3.8. If $(p, q) \Vdash \phi\left(\sigma_{\sim}, \ldots, \sigma_{n}\right)$ where $\sigma_{\sim}, \ldots, \sigma_{n}$ are canonical

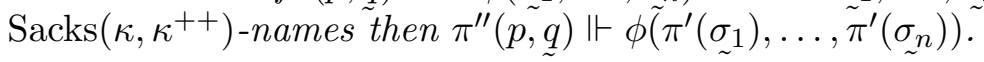

Notice that any $\operatorname{Sacks}\left(\kappa, \kappa^{++}\right)$-name can be viewed canonically as a $\operatorname{Sacks}\left(\kappa, \kappa^{++}\right) * \operatorname{Pr}(\underset{\sim}{U})$-name.

For contradiction, choose a condition $(p, q) \Vdash$ for all $\gamma \in Y, A_{\gamma} \in V^{\prime}$ and $(p, q) \in G_{\kappa}$ where $G_{\kappa}$ is the $\mathbb{P}_{\kappa}$-generic generated by $G_{\kappa}^{S}$ and $C$.

The $\operatorname{Sacks}\left(\kappa, \kappa^{++}\right)$-name $\underset{\sim}{q}$ is a name for a Prikry condition $(s, \underset{\sim}{A})$ where $s$ is a finite sequence of elements of $\kappa$ (and therefore can be assumed to be in the "ground model" $V_{0}$ ) and $\underset{\sim}{A}$ is a name for a subset of $\kappa$ in $\underset{\sim}{U}$. We may build a generalised fusion sequence below $p$ with meet $p^{\prime}$ such that $p^{\prime}$ will decide $\kappa$-many possibilities for $\underset{\sim}{A}$. 
The name $\underset{\sim}{C}$ is a $\operatorname{Sacks}\left(\kappa, \kappa^{++}\right) * \operatorname{Pr}(\underset{\sim}{U})$-name for a subset of $\kappa$ of size $\aleph_{0}$. We may extend $\left(p^{\prime}, q^{\prime}\right)$ to $\left(p^{\prime \prime}, q^{\prime \prime}\right)$ which decides $\kappa$-many possibilities for where the antichains in $\underset{\sim}{C}$ meet the generic. Let $Z^{*}$ be $X$ together with the support of $p^{\prime \prime}$, which has size $\kappa$. Rename $\left(p^{\prime \prime}, q^{\prime \prime}\right)$ to $(p, q)$, that is, we may assume without loss of generality that $(p, q)$ already had these properties.

Now let $i \in Y \backslash Z^{*}$ and $j>\gamma$ where $\gamma \in T$ is such that $\gamma>\sup \left(Z^{*}\right)$. Let $\pi$ be the automorphism which switches the Sacks component $i$ with the component $j$.

Now by Fact 3.8 , we have

$$
\pi^{\prime \prime}(p, \underset{\sim}{q}) \Vdash \pi^{\prime}\left(G_{\sim}^{S}(\kappa)\right)\left\lceilY \in V _ { \alpha ^ { * } } \left[\pi^{\prime}(\underset{\sim}{C}), \pi^{\prime}\left(G_{\sim}^{S}(\kappa)\right)\lceil X] .\right.\right.
$$

However, by choice of $Z^{*}$ we have $\pi^{\prime}(q)=q$ and $\pi(p)=p$. Also $G_{\sim}^{S}(\kappa)\lceil X$ and $\underset{\sim}{C}$ are fixed by $\pi^{\prime}, \pi^{\prime \prime}$, respectively, so in fact

$$
(p, \underset{\sim}{q}) \Vdash \pi^{\prime}\left(G_{\sim}^{S}(\kappa)\right)\left\lceilY \in V _ { \alpha ^ { * } } \left[\underset{\sim}{C}, G_{\sim}^{S}(\kappa)\lceil X]\right.\right.
$$

and in particular

$$
(p, \underset{\sim}{q}) \Vdash \pi^{\prime}\left(g_{i}\right) \in V_{\alpha^{*}}\left[\underset{\sim}{C}, G_{\sim}^{S}(\kappa)\lceil X]\right.
$$

where $\pi^{\prime}\left({\underset{\sim}{i}}_{i}\right)={\underset{\sim}{j}}_{j}$.

Since the condition $(p, q)$ was chosen in $G_{\kappa}$, we have $g_{j} \in V^{\prime}$. This reduces to the first case. The proof of Lemma 3.6 is complete.

Thus, we may choose $\gamma \in Y$ such that $A_{\gamma} \notin V^{\prime}$. However, Lemma 2.6 also holds in this case, giving the contradiction. This finishes the proof of Theorem 3.3.

4. The case of $\aleph_{\omega+1}$. In this section, let $\operatorname{Coll}(\alpha, \beta)$ denote the standard Levy collapse forcing which collapses $\beta$ to $\alpha$.

TheOREM 4.1. Assume $V \vDash G C H$ and there exists $\kappa$ which is $(\kappa+2)$ hypermeasurable. There is a forcing $\mathbb{P}$ such that in $V^{\mathbb{P}}$ there is no universal $\aleph_{\omega+1}$-graph and $2^{\aleph_{\omega}}>\aleph_{\omega+1}$.

Proof. We use the Prikry collapse forcing first developed by Magidor in [10] and put to further use by Gitik in [5. This forcing takes $\kappa$ as in the statement, increases $2^{\kappa}$ to $\kappa^{++}$and then collapses $\kappa$ to $\aleph_{\omega}$.

The proof is similar to that of Theorem 3.3 with the notable exception of Lemma 3.4.

Let $\mathbb{P}=\left\langle\mathbb{P}_{\alpha}: \alpha \leq \kappa\right\rangle$ be an Easton support iteration which is trivial except when $\alpha$ is inaccessible. For $\alpha \leq \kappa$ inaccessible let $\mathbb{P}_{\alpha}=\operatorname{Sacks}\left(\alpha, \alpha^{++}\right)$.

Let $G_{\kappa}^{S}$ be generic for $\operatorname{Sacks}\left(\kappa, \kappa^{++}\right)$over $V[G(<\kappa)]=V_{0}$. For $\alpha \leq \kappa^{++}$ we denote $V\left[G(<\kappa), G_{\kappa}^{S}(<\alpha)\right]$ by $V_{\alpha}$. For each component $\gamma$ of $\operatorname{Sacks}\left(\kappa, \kappa^{++}\right)$, we denote by $A_{\gamma}$ the subset of $\kappa$ which codes the generic for that component. 
By arguments in [2], the forcing $\mathbb{P}$ preserves cardinals and the measurability of $\kappa$ and sets $2^{\kappa}=\kappa^{++}$. If $j^{\prime}: V \rightarrow M$ witnesses the $(\kappa+2)$ hypermeasurability of $\kappa$ in $V$ then let $j: V_{\kappa^{++}} \rightarrow M[H]$ be the elementary embedding generated by the ultrapower on $U$, a normal measure on $\kappa$ in $V_{\kappa^{++}}$. We need to show that $\mathbb{P}$ has the additional property that in the $\mathbb{P}$-generic extension $V_{\kappa^{++}}$there exists a set $\mathcal{G}$ which is generic for $\mathrm{Coll}^{M[H]}\left(\left(\kappa^{+++}\right)^{M[H]}, j(\kappa)\right)$ over $M[H]$. This is called the guiding generic and is used to ensure that the Prikry collapse forcing has the $\kappa^{+}$-cc. The existence of the guiding generic after such Sacks forcing has been shown and will appear in A. Halilović's forthcoming $\mathrm{PhD}$ thesis. We provide the proof here for the convenience of the reader.

Lemma 4.2. There exists a set $\mathcal{G} \in V_{\kappa^{++}}$which is generic for $C M=$ $\mathrm{Coll}^{M[H]}\left(\left(\kappa^{+++}\right)^{M[H]}, j(\kappa)\right)$ over $M[H]$.

Proof. Let $H(<j(\kappa))$ be the generic for the forcing $j(\mathbb{P})$ in $M$ up to $j(\kappa)$. We know that $C M$ has the $j(\kappa)$-cc in $M[H]$ and moreover that $\operatorname{Sacks}^{M[H(<j(\kappa))]}\left(j(\kappa), j(\kappa)^{++}\right)$is $(<j(\kappa))$-closed. Thus, for any $A \subseteq C M$ a maximal antichain, $A \subseteq M[H(<j(\kappa))]$.

Now $A=\sigma^{M[H(<j(\kappa))]}$ where $\sigma$ is a $j(\mathbb{P}(<\kappa))$-name in $M$. We see that $[\sigma=j(f)(\alpha)]^{\tilde{H}(<j(\kappa))}$ for some $\alpha \tilde{<}\left(\kappa^{++}\right)^{M}$ and $f \in V_{0}$ such that $f: \kappa \rightarrow V$. Since $|j(\mathbb{P}(<\kappa))|=j(\kappa)$ and $j(\mathbb{P}(<\kappa))$ has the $j(\kappa)$-cc (as $\kappa$ is inaccessible), we have $j(f)(\alpha) \in V_{j(\kappa)}$ and so we may assume $f: \kappa \rightarrow V_{\kappa}$.

For each $f: \kappa \rightarrow V_{\kappa}$, define $F_{f}=\left\{A \subseteq C M: A=j(f)(\alpha)^{H(<j(\kappa))}\right.$ for some $\left.\alpha<\left(\kappa^{++}\right)^{V}\right\}$. Then $\left|F_{f}\right|=\left(\kappa^{++}\right)^{M}$ and $C M$ is $\kappa^{+++}$-distributive in $M[H(<j(\kappa))]$. Therefore, for each $f$ we may find a condition $p_{f} \in C M$ which meets every antichain in $F_{f}$. Since there are only $2^{\kappa}=\kappa^{+}$-many functions $f$ in $V$, we may use the $p_{f}$ 's as a base for forming the generic filter.

Using the guiding generic $\mathcal{G}$ and a normal measure $U$ on $\kappa$ we may now define Prikry collapse forcing $R$ in $V_{\kappa^{++}}$. A condition $p \in R$ is a tuple $(n, \bar{\alpha}, \bar{f}, A, F)$ such that the following hold:

- $n<\omega$,

- $\bar{\alpha}=\left\langle\alpha_{i}: i<n\right\rangle$ is a strictly increasing sequence of inaccessibles such that $\alpha_{0}=\aleph_{1}$,

- $\bar{f}=\left\langle f_{i}: i<n\right\rangle$ is such that for $i<n-1$ we have $f_{i} \in \operatorname{Coll}\left(\alpha_{i}^{+++}, \alpha_{i+1}\right)$ and $f_{n-1} \in \operatorname{Coll}\left(\alpha_{n-1}^{+++}, \kappa\right)$,

- $A \in U$ is such that $\min (A)>\alpha_{n-1}$,

- $\operatorname{dom}(F)=A$ and $F(\alpha) \in \operatorname{Coll}\left(\alpha^{+++}, \kappa\right)$ for all $\alpha \in A$,

- $[F]_{U}$ which is in $\mathrm{Coll}^{M}\left(\kappa^{+++}, j(\kappa)\right)$ is an element of $\mathcal{G}$.

The conditions are ordered as follows: we have $p^{\prime} \leq p$ if and only if

- $n^{\prime} \geq n$,

- for all $i<n$ we have $\alpha_{i}=\alpha_{i}^{\prime}$ and $f_{i} \subseteq f_{i}^{\prime}$, 
- $A^{\prime} \subseteq A$,

- if $\alpha \in A$ then $F(\alpha) \subseteq F^{\prime}(\alpha)$,

- for all $i \geq n$ we have $\alpha_{i}^{\prime} \in A$ and $f_{i}^{\prime} \supseteq F\left(\alpha_{i}^{\prime}\right)$.

Prikry collapse forcing has the $\kappa^{+}$-cc.

Fix $U, \mathcal{G} \in V_{\kappa^{++}}$the normal measure on $\kappa$ and the guiding generic which are used to define the Prikry collapse forcing. As in the proof of Theorem 3.3 , we must be able to restrict the Sacks product at $\kappa$, but still be able to define the Prikry collapse generic. To do this, we first need that the Prikry collapse generic is completely determined by the $\omega$-sequence of collapses.

LEMmA 4.3. If $C=\left\langle G_{\alpha_{i}}: i\langle\omega\rangle\right.$ is the sequence of generics for the Levy collapses $\operatorname{Coll}\left(\alpha_{i}^{+++}, \alpha_{i+1}\right)$ which are derived from the Prikry collapse generic $G^{R}$ then $G^{R}$ is uniquely determined by $C$.

Proof. Let $\bar{\alpha}_{\omega}$ be the $\omega$-sequence of ordinals $\alpha_{i}$ from $C$. Let $S$ be the set of conditions in Prikry collapse forcing $(n, \bar{\alpha}, \bar{f}, A, F)$ such that

- $n<\omega$,

- $\bar{\alpha}=\left\langle\alpha_{i}: i<n\right\rangle$ is an initial segment of $\bar{\alpha}_{\omega}$,

- $\bar{f}=\left\langle f_{i}: i<n\right\rangle$ is such that for $i<n-1$ we have $f_{i} \in G_{\alpha_{i}}$ and $f_{n-1} \in \operatorname{Coll}\left(\alpha_{n-1}^{+++}, \kappa\right)$,

- $A \in U$ is such that $\min (A)>\alpha_{n-1}$ and $\left\{\alpha_{i} \in \bar{\alpha}_{\omega}: i \geq n\right\} \subseteq A$,

- $\operatorname{dom}(F)=A$ and $F(\alpha) \in \operatorname{Coll}\left(\alpha^{+++}, \kappa\right)$ for all $\alpha \in A$,

- for $i \geq n$ we have $F\left(\alpha_{i}\right) \in G_{\alpha_{i}}$,

- $[F]_{U}$ which is in $\mathrm{Coll}^{M}\left(\kappa^{+++}, j(\kappa)\right)$ is an element of $\mathcal{G}$.

We will show that any two conditions $p, p^{\prime}$ in $S$ are compatible. Assume without loss of generality that $n \leq n^{\prime}$. The only problem in finding a condition below these two is that $F$ and $F^{\prime}$ might contain incompatible conditions from the collapses. However, $j(F)(\kappa)$ and $j\left(F^{\prime}\right)(\kappa)$ are both elements of $\mathcal{G}$ and so must be compatible. Thus there is a set $B \in U$ such that for $\alpha \in B$ we have $\alpha \in A \cap A^{\prime}$ and $F(\alpha)$ and $F^{\prime}(\alpha)$ are compatible. Now let $F^{\prime \prime}$ have domain $B \cup\left\{\alpha_{i}: i \geq n\right\}$ such that for $\alpha \in B$ we have $F^{\prime \prime}(\alpha)<F(\alpha), F^{\prime}(\alpha)$.

Since $f_{i}$ and $f_{i}^{\prime}$ both come from the generic $G_{\alpha_{i}}$, they must also be compatible. Let $f_{i}^{\prime \prime}<f_{i}, f_{i}^{\prime}$ for $i \leq n-1$, let $f_{i}^{\prime \prime}=f_{i}^{\prime}$ for all $i \in\left[n, n^{\prime}-1\right)$ and let $f_{n-1}^{\prime \prime}<f_{n-1}^{\prime}, F^{\prime \prime}\left(\alpha_{n-1}^{\prime}\right)$. Call this $\bar{f}^{\prime \prime}=\left\langle f_{i}^{\prime \prime}: i<n^{\prime}\right\rangle$.

Now let $p^{\prime \prime}=\left(n^{\prime}, \bar{\alpha}^{\prime}, \bar{f}^{\prime \prime}, B, F^{\prime \prime}\right)$. It is routine to check that this is a condition and below $p$ and $p^{\prime}$.

It is also easy to check that $G^{R} \subseteq S$. Therefore, since any condition compatible with all conditions in the generic is in the generic, we have $G^{R}=S$.

Lemma 4.4. The set $T$ of all $\alpha<\kappa^{++}$with $\operatorname{cf}(\alpha)=\kappa^{+}$such that $U \cap V_{\alpha}$ $\in V_{\alpha}$ and for which $C$ (as in Lemma 4.3) determines a Prikry collapse generic over $V_{\alpha}$ is a stationary subset of $\kappa^{++}$. 
Proof. Let $U$ be a $\mathbb{P}$-name for $U$, let $\mathcal{G}$ be a $\mathbb{P}$-name for $\mathcal{G}$ and let $\bar{N} \in V$ be an elementary submodel of some large $H(\theta)$ which has size $\kappa^{+}$, is closed under $\kappa$-sequences and has $\mathbb{P}, U$ and $\mathcal{G}$ as elements. Let $N$ be the transitive collapse of $\bar{N}$. By closure under $\kappa$-sequences we deduce that $\alpha=N \cap \kappa^{++}$ has cofinality $\kappa^{+}$. In fact, since $\kappa^{+} \subseteq N$, it follows that $H\left(\kappa^{++}\right) \cap N$ is transitive.

Similar arguments to Lemma 3.4 show that $N[G(<\alpha)]$ is an elementary submodel of $H(\theta)[G]$.

By the generalised fusion property of $\kappa$-Sacks forcing (which implies $\kappa$ covering) and arguments as in Lemma 3.4, any subset of $\kappa$ in $V_{\alpha}$ has a name of size $\kappa$ in $\mathbb{P}(<\alpha)$ and therefore has a name in $N$. It follows by elementarity that $U^{G} \cap N[G(<\alpha)]$ equals $U^{G(<\alpha)}$ and belongs to $N[G(<\alpha)]$, therefore to $V_{\alpha}$, as desired.

Let $\pi: N[G(<\alpha)] \rightarrow H(\theta)[G]$ be the lifted embedding. We will show that if $H$ is Prikry collapse generic over $H(\theta)$ using $U$ and $\mathcal{G}$, then $\pi^{-1}[H]$ is Prikry collapse generic using $\pi^{-1}(U)=U \cap N[G(<\alpha)]$ and $\pi^{-1}(\mathcal{G})$.

First note that $\pi^{-1}(U)=U \cap V_{\alpha}$ since $N[G(<\alpha)]$ and $V_{\alpha}$ have the same subsets of $\kappa$. Also $\pi^{-1}(\mathcal{G})$ is a subset of $\operatorname{Coll}\left(\kappa^{+++}, j(\kappa)\right)$ in the ultrapower by $U \cap N[G(<\alpha)]=U \cap V_{\alpha}$ and thus also belongs to $V_{\alpha}$.

Let $X \in N[G(<\alpha)]$ be a maximal antichain in $R^{N[G(<\alpha)]}\left(\pi^{-1}(U), \pi^{-1}(\mathcal{G})\right)$. Then $\pi(X)$ is a maximal antichain in $R(U, \mathcal{G})$. By the $\kappa^{+}$-cc, $|X| \leq \kappa$. The critical point of $\pi$ must be $>\kappa^{+}$since $N$ is closed under $\kappa$-sequences, so $\pi(X)=\pi[X]$.

Let $C$ be the $\omega$-sequence generated by the generic as in Lemma 4.3. By the same lemma, $C$ is uniquely determined by $H$. Using the same reasoning as for $\pi(X)$, we also have $\pi(C)=\pi[C]$. Therefore,

$$
V_{\alpha}[G(<\alpha)]\left[\pi^{-1}[H]\right]=V_{\alpha}[G(<\alpha)][C] .
$$

For $\alpha \in T$ it is clear that $U \cap V_{\alpha}$ is an ultrafilter in $V_{\alpha}$ and $\mathcal{G} \cap V_{\alpha}$ is a guiding generic in $V_{\alpha}$ so we may define the Prikry collapse forcing $R\left(U \cap V_{\alpha}, \mathcal{G} \cap V_{\alpha}\right)$ in $V_{\alpha}$. Let $C$ be the Prikry collapse generic $\omega$-sequence over $V_{\kappa^{++}}$. By Lemma 4.3 , for $\alpha \in T$ we deduce that $C$ is also the generic $\omega$-sequence for $R\left(U \cap V_{\alpha}, \mathcal{G} \cap V_{\alpha}\right)$ over $V_{\alpha}$.

The rest of the argument proceeds as in the proof of Theorem 3.3 using the fact that Prikry collapse forcing has the $\kappa^{+}$-cc.

Assume that $H^{*} \in V_{\kappa^{++}}[C]$ is a universal graph of size $\kappa^{+}$and let $\alpha^{*} \in T$ be such that $H^{*} \in V_{\alpha^{*}}[C]$ as in Lemma 2.3 .

As in Counterexample Construction 2.4 construct the graph $H \in V_{\kappa^{++}}$ with universe $\kappa \cup Y$ where $Y \subseteq \kappa^{++}$is of size $\kappa^{+}$with $\min (Y)>\alpha^{*}$ and $Y \cap T$ is cofinal in $\sup (Y)$ and has cofinality $\kappa^{+}$. 
By the universality of $H^{*}$ there exists an embedding $f: H \rightarrow H^{*}$. Find $V^{\prime}$ and $X$ in a similar way to Lemma 3.5 so that $f\left\lceil\kappa \in V^{\prime}\right.$. To see that there is a $\beta \in Y \backslash X$ such that $A_{\beta} \notin V^{\prime}$, we need the analogue of Lemma 3.6 .

The proof of Lemma 3.6 relies on properties of the Sacks forcing except when showing that it cannot be the case that $V_{\gamma}\left[A_{\beta}\right] \subseteq V_{\gamma}[C]$. By Claim 3.7. $V_{\gamma}\left[A_{\beta}\right]$ contains a club in $\kappa^{+}$which does not contain a club in $V_{\gamma}$ and again the Prikry collapse forcing has the $\kappa^{+}$-cc and therefore all clubs in $V_{\gamma}[C] \cap \kappa^{+}$contain clubs in $V_{\gamma}$.

Thus, we may choose $\gamma \in Y$ such that $A_{\gamma} \notin V^{\prime}$. As Lemma 2.6 also holds, we obtain the desired contradiction. This finishes the proof of Theorem 4.1.

5. High complexity. In fact, the forcings above give even stronger negative universality results. We proved in the cases above that there cannot be one $\lambda^{+}$-graph which embeds all $\lambda^{+}$-graphs, but it is also true that no small family of such graphs can embed the rest.

The following is an analogue of Theorem 2.2 .

THEOREM 5.1. Let $V \vDash \lambda^{<\lambda}=\lambda$ and let $P$ be $a \leq \lambda$-support product forcing $\left\langle P_{\alpha}: \alpha<\alpha^{*}\right\rangle$ such that $\alpha^{*} \geq \lambda^{+++}$is regular and $P$ has the $\lambda$-covering property and such that the generic at any component $P_{\alpha}$ can be coded as a subset of $\lambda$. Then in $M^{P}$ the complexity for $\lambda^{+}$-graphs is $2^{\lambda}=\alpha^{*}$.

Note that if there is no universal $\lambda^{+}$-graph, the minimum complexity must be $\geq \lambda^{++}$as the disjoint union of a $\leq \lambda^{+}$-sized universal family would be a universal $\lambda^{+}$-graph. This is the reason that we only consider products of length $\geq \lambda^{+++}$.

Proof of Theorem 5.1. For contradiction let $\bar{H}=\left\{H_{\beta}: \beta<\beta^{*}\right\}$ for some $\beta^{*}<\alpha^{*}$ be a small universal family of $\lambda^{+}$-graphs in $V^{P}$. By similar arguments to Theorem 2.2 we know that $\bar{H}$ must be caught by some initial segment of the product of length $\gamma<\alpha^{*}$. Define the graph $H$ as in Counterexample Construction 2.4 where $Y=\left[\gamma, \gamma+\lambda^{+}\right)$. By assumption there is $\beta$ such that there is an embedding $f: H \hookrightarrow H_{\beta}$. The rest of the argument proceeds as before.

The analogue to Theorem 3.3 requires slightly more consistency strength to extend the product. Namely, for a product of length $\kappa^{(+\alpha)}$, we require that $\kappa$ be $(\kappa+\alpha)$-hypermeasurable (see [5]).

TheOREM 5.2. Assume $V \vDash G C H$ and $\kappa$ is a $(\kappa+\alpha)$-hypermeasurable cardinal for some ordinal $\alpha$ such that $\kappa^{+\alpha}$ is regular. There is a forcing $P$ such that in $V^{P}$ the complexity of $\kappa^{+}$-graphs is $2^{\kappa}=\kappa^{+\alpha}$ and $\kappa$ is singular.

Note that the proof of this requires a straightforward generalisation of Lemma 3.4 to longer products. 
6. Open questions. We conclude with some possible continuations on this subject.

Question 6.1. Is it possible to extend this type of proof to get the non-existence of a universal graph at the successor of a singular of cofinality $>\aleph_{0} ?$

Question 6.2. In the case where a measurable is collapsed to $\aleph_{\omega}$, how far can we raise the powerset of $\aleph_{\omega}$ and still obtain the negative universality result? What if it were collapsed to $\aleph_{\omega_{1}}$ ?

Acknowledgements. The first author was supported by Grant P 19375N18 of the Austrian Science Fund (FWF).

The second author was supported by Lise-Meitner Project number M1076-N13 from the FWF.

Both authors would like to thank the referee for many helpful comments and suggestions.

\section{References}

[1] M. Džamonja and S. Shelah, Universal graphs at the successor of a singular cardinal, J. Symbolic Logic 68 (2003), 366-388.

[2] S. Friedman and K. Thompson, Perfect trees and elementary embeddings, ibid. 73 (2008), 906-918.

[3] M. Gitik, The negation of the singular cardinal hypothesis from $o(\kappa)=\kappa^{++}$, Ann. Pure Appl. Logic 43 (1989), 209-234.

[4] - , The strength of the failure of the singular cardinal hypothesis, ibid. 51 (1991), $215-240$.

[5] —, On measurable cardinals violating the continuum hypothesis, ibid. 63 (1993), 227-240.

[6] T. Jech, Set Theory, 3rd Millennium Edition, Springer Monogr. Math., Springer, 2003.

[7] A. Kanamori, Perfect-set forcing for uncountable cardinals, Ann. Math. Logic 19 (1980), 97-114.

[8] M. Kojman and S. Shelah, Nonexistence of universal orders in many cardinals, J. Symbolic Logic 57 (1992), 875-891.

[9] K. Kunen, Set Theory, Stud. Logic Found. Math. 102, North-Holland, 1980.

[10] M. Magidor, On the singular cardinals problem I, Israel J. Math. 28 (1977), 1-31.

[11] A. Mekler, Universal structures in power $\aleph_{1}$, J. Symbolic Logic 55 (1990), 466-477.

[12] S. Shelah, On universal graphs without instances of $\mathrm{CH}$, Ann. Pure Appl. Logic 26 (1984), 75-87.

[13] —, Universal graphs without instances of CH: revisited, Israel J. Math. 70 (1990), 69-81. 
[14] K. Thompson, Universality for orders and graphs which omit large substructures, Notre Dame J. Formal Logic 47 (2006), 233-248.

S.-D. Friedman

Kurt Gödel Research Center for Mathematical Logic

Währinger Straße 25

A-1090 Wien, Austria

E-mail: sdf@logic.univie.ac.at

http://www.logic.univie.ac.at/ sdf/
K. Thompson Institut für Diskrete Mathematik und Geometrie Technische Universität Wien Wiedner Hauptstraße 8-10/104 A-1040 Wien, Austria E-mail: katherine.thompson@tuwien.ac.at http://www.logic.univie.ac.at/ thompson

Received 10 June 2009;

in revised form 16 June 2010 